\title{
Successful management of a rare case of giant extra- abdominal fibromatosis
}

\begin{abstract}
Fibromatosis or desmoid tumor is a rare a locally aggressive neoplasm with variable anatomic location and clinical course. Due to its rarity and diversity, to date, there is no a gold standard approach and the main and more update evidence regard mainly the high-income countries. We presented a rare case of giant extra-abdominal fibromatosis successfully treated in a low-income setting.
\end{abstract}

Keywords: fibromatosis, desmoid tumor, extra-abdominal fibromatosis, low-income countries
Volume IO Issue I - 2020

\author{
Mario Antunes,' Damiano Pizzol, ${ }^{2}$ Anna \\ Claudia Colangelo ${ }^{2,3}$ \\ 'Department of Surgery, Central Hospital of Beira, Beira, \\ Mozambique \\ ${ }^{2}$ Operational Research Unit, Doctors with Africa Cuamm, \\ Mozambique \\ ${ }^{3}$ Department of Surgery and Organ Transplantation, University \\ of Padua, Italy
}

\author{
Correspondence: Damiano Pizzol, Operational Research \\ Unit, Doctors with Africa CUAMM, Mozambique, Tel (+39) \\ 366873|237, Email d.pizzol@cuamm.org
}

Received: November 13, 2019 | Published: January 22, 2020

\section{Introduction}

Aggressive fibromatosis, also known as desmoid tumor or desmoid-type fibromatosis are defined by the World Health Organization (WHO), as clonal fibroblastic proliferation that arises in the deep soft tissues and is characterized by infiltrative growth and a tendency toward local recurrence but an inability to metastasize. ${ }^{1}$ Fibromatosis has variable and often unpredictable anatomic locations clinical course, and biologic behaviors. ${ }^{2}$ Its incidence is five to six cases per 1 million of the population per annum and, due to the rarity of this disease, a very low level of evidence is present in the scientific literature. ${ }^{2}$ Although it is a histologically benign neoplasm, in high income country, the sole surgery approach does not represent the gold standard as it is poorly encapsulated and locally invasive. ${ }^{3}$ Indeed, growing evidence suggests a multidisciplinary and tailored approach including radiation therapy, hormonal therapy, or cytotoxic chemotherapy and considering that most patients can be managed with one treatment modality at a time. ${ }^{2}$

However, in low-resources settings, a multidisciplinary approach is unlikely both due to the lack of specialized health workers and the absence of adequate equipment and drugs for diagnosis, therapy and follow-up. We reported a case of a rare case of giant extra-abdominal fibromatosis successfully treated in a low-income setting.

\section{Case report}

A 25-year-old woman presented in our institution, with a back giant mass, evolved over about two years (Figure 1 A \& B). The patient had no pain or other symptoms related to the mass. History of previous diseases was unremarkable, her general condition was good, and she had normal vital parameters. It was not possible to perform a magnetic resonance imaging and we decided to hospitalize the woman for surgery with a diagnosis of possible fibroma. She was HIV negative, and preoperative tests showed normal parameters.

The patient underwent surgical excision: we performed a diamond engraving in the middle on the mass. We tried to remove the entire tumor but it was poorly encapsulated with an extensive vascular supply. After hemostasis, in order to close the skin, we performed a slip flap.
The excised mass was $25 \times 17 \times 12 \mathrm{~cm}$. Histologic examination showed sweeping fascicles of myofibroblasts with abundant collagenous stroma and the diagnosis was fibromatosis. The post surgical course was regular and the 3-month follow-up showed a clean scar (Figure $1 \mathrm{C})$ and no sequela.

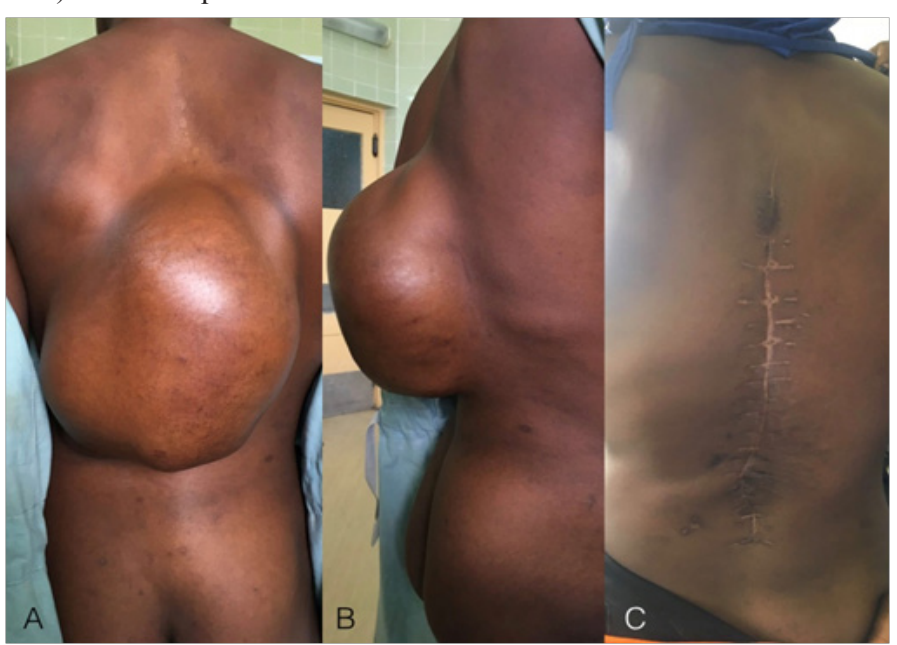

Figure I Giant fibromatosis at presentation (A and $\mathrm{B}$ ) and three months after surgery $(C)$.

\section{Discussion}

A recent consensus algorithm on fibromatosis suggests a multidisciplinary approach in order to obtain the best tailored management for patients affected by this disease. ${ }^{2}$ Compared to this algorithm, the management of our case is very far in diagnostic, treatment and follow up procedures. Magnetic Resonance Imaging (MRI) is considered the gold standard of imaging in fibromatosis, not only for diagnosis, but also for staging and follow-up. Unfortunately, our hospital is not provided of MRI nor CT-scan and also ultrasound were not available. Moreover, other useful tests are biopsy, molecular biology and genetic. Among these, the only possible would have been the biopsy for an histological examination but we avoid the biopsy and used a sample of the excised lump for the histologic diagnosis. 
With hindsight, although the surgery and the overall management was successful, to perform a biopsy should be recommended in order to help in assessing the risks and benefits of surgery or other therapies, including doing nothing. Thus, the decision to subject the patient to surgery was driven on one hand by the necessity to remove the giant lump and, on the other hand, by the lack of other choices. When possible, the consensus algorithm suggest a multidisciplinary and tailored management in agreement with the patient andconsidering alternative or adjuvant options as radiation therapy, hormonal therapy, or cytotoxic chemotherapy. ${ }^{2}$

Thees observations highlight the weakness of low-income countries health systems and suggest the development of algorithm consensus addressed to this poor settings. Especially for rare diseases, in fact, it is crucial, for the few and overload health professional to have clear, applicable and effectiveness guide line. In particular, although we have described just a case, we point out at least three recommendation: 1) to pay attention to alert signs (masses greater than $3-5 \mathrm{~cm}$, deep located, rapid growth), 2) to use radiologic adequate imaging, as ultrasound, both in order to make differential diagnosis (i.e. hematoma, abscess, infections) and to planning biopsy and surgery; 3 ) to perform the biopsy in order to help in assessing the risks and benefits of surgery or other therapies.

In this case, also looking at the three months follow-up, we can consider satisfactory the management of this case. However, we don't know the risk of relapse and, likely, in few months we will lose on follow-up the patient. To avoid this, not only in case of fibromatosis but in oncology in general, it is mandatory to strengthen the health system with particular attention to physical, psychologic and social aspects and to promote comprehensive programs including physical, psychologic and social support.

\section{Ethic statement}

Written informed consent was obtained from the patient for publication of this case report and any accompanying images.

\section{Acknowledgments}

None.

\section{Funding}

None.

\section{References}

1. Fletcher CDM, Bridge JA, Hogendoorn P, et al. WHO Classification of Tumours of Soft Tissue and Bone. 4 ed, Lyon: IARC. 2013.

2. Kasper B, Baumgarten C, Garcia J, et al. Anupdate on the management of sporadic desmoid-type fibromatosis: a European Consensus Initiative between Sarcoma PAtients EuroNet (SPAEN) and European Organization for Research and Treatment of Cancer (EORTC)/Soft Tissue and BoneSarcoma Group (STBSG). Ann Oncol. 2017;28(10):2399-2408.

3. Chu KF, Samara MS, Laver N, Wein RO. A rare presentation of a largeextra-abdominal desmoid tumor of the posterior neck and back. $\mathrm{Am}$ J Otolaryngol. 2013 Nov-Dec;34(6):727-730. 